\title{
Numerical models of advanced ceramic absorbers for volumetric solar receivers
}

\author{
Raffaele Capuano $^{\mathrm{a}, *}$, Thomas Fend ${ }^{\mathrm{b}}$, Peter Schwarzbözl ${ }^{\mathrm{b}}$, Olena Smirnova ${ }^{\mathrm{b}}$, \\ Hannes Stadler ${ }^{\mathrm{a}}$, Bernhard Hoffschmidt ${ }^{\mathrm{b}}$, Robert Pitz-Paal ${ }^{\mathrm{b}}$ \\ a Institute of Solar research, German Aerospace Center (DLR), Professor-Rehm-Str. 1, 52428 Jülich, Deutschland \\ ${ }^{\mathrm{b}}$ Institute of Solar research, German Aerospace Center (DLR), Linder Höhe, 51147 Köln, Deutschland
}

\section{A R T I C L E I N F O}

\section{Article history:}

Received 13 November 2014

Received in revised form

28 August 2015

Accepted 16 December 2015

\section{Keywords:}

Volumetric solar receiver

CSP

Numerical simulation

Analytical model

\begin{abstract}
A B S T R A C T
Nowadays, due to the pressing global energy demand, a possible source of renewable energy is represented by solar energy. Concentrated solar power (CSP) represents an excellent alternative, or add-on to existing systems for large-scale energy production.

In those systems and, particularly, in solar tower plants (STP), mirroring surfaces reflect sunlight, focusing it on the core-component defined as volumetric receiver. Here, a porous structure (absorber) is responsible for converting the solar radiation into heat with the use of evolving air under natural convection.

The proper design of this element is essential in order to achieve high efficiencies that can make such facilities extremely advantageous.

In this paper, an overview of the different approaches for the numerical modeling developed at the German Aerospace Center (DLR), are presented, based both on detailed and homogeneous representation (discrete and continuum approaches, respectively) of the absorber.

The corresponding numerical models were run again and compared where possible, using as reference case pre-exiting experimental evaluation, pointing out shortcomings and differences.

Temperature profiles for solid and fluid phase, as well as efficiency value, are used as key parameters in the comparison, showing a substantial gap between numerical and experimental results.

This inconsistency can be addressed both to inherent difficulties in the experiments butalso on the lack of a proper characterization of the absorber radiative behavior that directly affects the heat transfer process. This gap is even more pronounced when the use of a continuum approach is considered, due to implicit approximation introduced by the homogeneous representation of the porous volume. For those reasons, a better representation of the radiative heat transfer must be introduced in future conjugate numerical models and the use of a continuum approach can be addressed to a preliminary design procedure, given the possibility to quickly manipulate geometric and thermodynamic parameters of porous structures.
\end{abstract}

(c) 2015 Elsevier Ltd. All rights reserved.

\section{Contents}

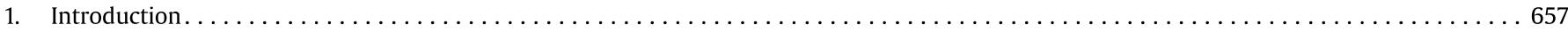

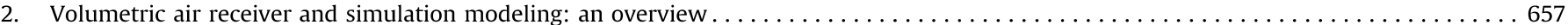

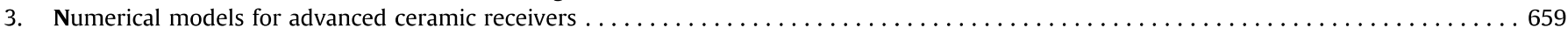

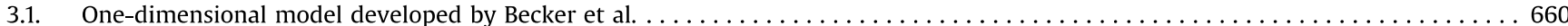

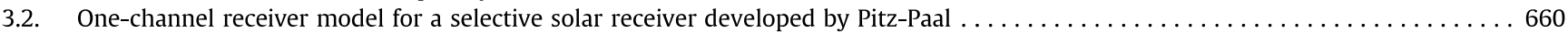

3.3. Quasi-three-dimensional analytical model for a volumetric absorber structure developed by Hoffschmidt .................... 661

3.4. Discrete and continuum approach for the investigation of flow through volumetric receiver developed by Fend and Smirnova .... 662

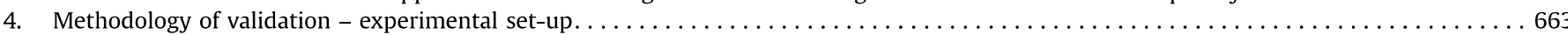

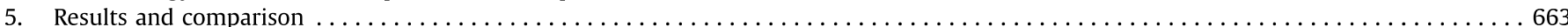

\footnotetext{
* Corresponding author. Tel.: +492203601 4175; fax: +4922036014170.

E-mail address: raffaele.capuano@dlr.de (R. Capuano).
} 


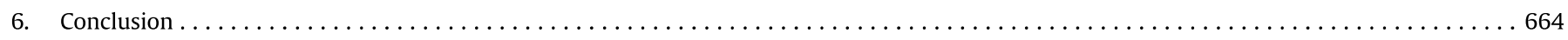

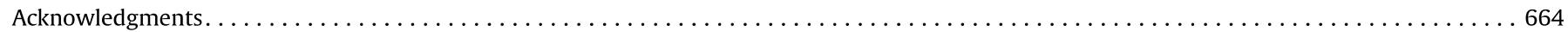

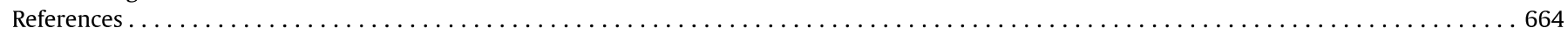

\section{Introduction}

In the current climate of growing energy needs and increasing environmental concern, alternatives to the use of fossil fuels have to be investigated. One such alternative is solar energy.

The radiation coming from the sun is a very high-quality energy source due to the high temperatures reached and the exergy content of its source. Nevertheless, only a small fraction of this energy reaches the earth producing a total flux too small to reach high temperature levels; thus, optical concentration systems are required [1].

One of the most likely candidates for providing the majority of solar renewable energy is the concentrated solar power (CSP), used for electricity production. Those systems use mirrored panels or lenses to concentrate the solar radiation at a focal point or line. The concentrated light is then converted into heat, which drives a heat engine connected to an electrical power generator.

The technical feasibility of CSP has been considered sufficiently mature since the demonstration plants built mostly during the 1980 s [2-5], and a wide variety of receivers has been tested in either numerical or experimental way.

CSP plants produce electricity in a similar way to conventional power stations, using steam to drive a turbine. The difference is that the energy comes from solar radiation and is converted into high-temperature heat, between 400 and $1000{ }^{\circ} \mathrm{C}$. Three main technologies have been identified during the past decades for generating electricity from $10 \mathrm{~kW}$ up to more than $300 \mathrm{MW}$ :

- dish/engine technology, which can directly generate electricity in isolated locations;

- parabolic and Fresnel trough technology;

- solar tower technology, in which high temperatures will be achieved and are also suitable for solar chemistry applications.

The solar radiation is then focused directly on another basic component: the solar receiver.

Several heat transfer fluids such as liquid sodium, saturated or super-heated steam, nitrate molten salts and air, have been tested in those plants. In today's context, receivers working with air as the heat transfer fluid are being considered a favorable option even if there are some points not fully solved, like absorber durability, receiver efficiency and the specific cost.

Nevertheless, the associated advantage of the air receiver, which allows to obtain higher temperature compared to receivers using liquids as evolving fluid, allows higher-efficiency thermodynamic cycles.

Two different types of solar receivers are available: linear receivers and volumetric receivers.

In linear receivers solar radiation is absorbed on the outer face of tubes traversed by the evolving fluid, depending upon the absorptive and reflective characteristics of the surface material. A low thermal conductivity and a large wall thickness lead to a high temperature at the outer surface, which again influences the emission of the tube and thus the thermal losses.

These disadvantages are partially solved by use of volumetric receivers. Here, the absorbed solar radiation penetrates as deep as possible into the absorber structure heating up the heat transfer fluid.
Another important advantage of volumetric receivers compared to a linear receiver, is that the volume mainly has a thermal function. While with linear receivers the wall temperature is higher than the fluid temperature, the use of volumetric receivers could allow the presence of the so called volumetric effect [6]. Due to the porous structure which absorbs the concentrated solar radiation and is simultaneously cooled by the heat transfer fluid, the front side of the receiver can stay cooler than the evolving medium.

Solar tower technology utilizes both linear and volumetric receivers for high temperature applications. The receiver is placed on the top of the tower where heliostats focus the incoming sunlight, which is then converted into heat.

At present, in Jülich, Germany, a 1.5 MWe pre-commercial demonstration power tower plant with ceramic volumetric receiver and thermal storage has been in operation since 2009 [7]. The continuous research and development in volumetric receivers make this technology a promising solar power alternative.

This paper is an overview of numerical approaches developed at the DLR and used for the simulation and the analysis of the behavior of volumetric receivers. The simulation of a porous material in terms of key-characteristics or behaviors leads to several improvements and understanding enhancements needed for the design of advanced material concepts to be used in the near future in solar thermal power plants.

\section{Volumetric air receiver and simulation modeling: an overview}

The central element of the volumetric receiver is the absorber, consisting of a three-dimensional structure with a certain volume on which the solar energy is concentrated; here the solar energy is then absorbed throughout the depth of this volume.

One of the key-parameters of an absorber structure is its porosity, $\varepsilon$. It is the fraction of the volume of voids over the total volume characterizing the structure.

Another important characteristic is the cell density, that defines how finely the material of the structure is divided. Further requirements are a high value of the porosity, in order to allow the concentrated solar radiation to penetrate into the volume of the cellular material, a high cell density to achieve large surface areas necessary to transfer heat from the material to the gaseous fluid flowing through the channels and a high thermal conductivity.

Since in high temperature solar applications values of above $800{ }^{\circ} \mathrm{C}$ are reached, ceramic materials are the most suitable option for this application but also metallic absorbers have been tested for the same application. The increased interest in ceramnic absorbers came out from the limitation set on the maximum outlet air temperature obtainable by metal absorbers. One of the most efficient setup, using an open loop volumetric receiver with metallic absorbers, was the one tested in the Phoebus-TSA (Technology Program Solar Air Receiver) project, a 2.5MWth volumetric air receiver together with a termal storage and steam generator with the ability to achieve an efficiency of $85 \%$ at an average flux of $0.3 \mathrm{MW} / \mathrm{m}^{2}$, producing steam at $500{ }^{\circ} \mathrm{C}$ with a stabilized outlet air temperature of $700{ }^{\circ} \mathrm{C}$. 


\begin{tabular}{|c|c|c|c|}
\hline \multicolumn{2}{|c|}{ Nomenclature } & $\zeta$ & pressure drop coefficient \\
\hline $\mathrm{q}$ & transferred energy rate $\left[\mathrm{W} \mathrm{m^{-2 } ]}\right.$ & $\varepsilon$ & porosity of absorber \\
\hline $\mathrm{q}_{0}$ & $\begin{array}{l}\text { heating power per unit volume for the conductive } \\
\text { heat transfer }\left[\mathrm{W} \mathrm{m}^{-3}\right]\end{array}$ & $\begin{array}{l}\xi \\
\Omega_{\mathrm{r} \varphi}\end{array}$ & $\begin{array}{l}\text { extinction coefficient of the radiation }\left[\mathrm{m}^{-1}\right] \\
\text { source term describing radial heat transport }\left[\mathrm{W} \mathrm{m}^{-3}\right]\end{array}$ \\
\hline Q & $\begin{array}{l}\text { heating power per unit volume for the convective heat } \\
\text { transfer }\left[\mathrm{W} \mathrm{m}^{-3}\right]\end{array}$ & $\varphi$ & $\begin{array}{l}\text { azimuth angle } \\
\text { dynamic viscosity }\left[\mathrm{kg} \mathrm{m}^{-1} \mathrm{~s}^{-1}\right]\end{array}$ \\
\hline A & area of element $\left[\mathrm{m}^{2}\right]$ & $\rho$ & density $\left[\mathrm{kg} \mathrm{m}^{-3}\right]$ \\
\hline$\dot{M}$ & mass flow rate of the heat transfer medium $\left[\mathrm{kg} \mathrm{s}^{-1}\right]$ & & \\
\hline $\mathrm{T}$ & $\begin{array}{l}\text { mass flow density }\left[\mathrm{kg} \mathrm{s}^{-1} \mathrm{~m}^{-2}\right] \\
\text { temperature }[\mathrm{K}]\end{array}$ & \multicolumn{2}{|c|}{ Superscripts } \\
\hline $\mathrm{Nu}$ & Nusselt number & - & receiver inlet \\
\hline $\operatorname{Re}$ & Reynolds number & " & receiver outlet \\
\hline $\operatorname{Pr}$ & Prandtl number & i & index of surface element \\
\hline \multirow{2}{*}{$\begin{array}{l}\mathrm{Pe} \\
\Delta \mathrm{T}_{\mathrm{g}}\end{array}$} & Peclet number & $\mathrm{j}$ & index of volume element \\
\hline & $\begin{array}{l}\text { bulk temperature difference between inlet and outlet } \\
\text { of a gas volume element }[\mathrm{K}]\end{array}$ & Subsc & \\
\hline$\Delta \mathrm{p}$ & pressure difference & & \\
\hline \multirow{2}{*}{$\begin{array}{l}Q_{i r} \\
A_{v}\end{array}$} & solar irradiated power $[\mathrm{W}]$ & in & inlet \\
\hline & heat transfer surface per volume $\left[\mathrm{m}^{2} \mathrm{~m}^{-3}\right]$ & out & outlet \\
\hline $\mathrm{z}$ & axial coordinate (solid and flow) & $\mathrm{O}$ & orifice \\
\hline$r$ & radial coordinate $[\mathrm{m}]$ & rec & receiver \\
\hline$c_{p}$ & specific heat capacity of the fluid $\left[\mathrm{W} \mathrm{kg}{ }^{-1} \mathrm{~K}^{-1}\right.$ ] & 1 & loss \\
\hline \multirow{2}{*}{$\begin{array}{l}\mathrm{p} \\
\mathrm{R}\end{array}$} & pressure $\left[\mathrm{N} \mathrm{m}^{-2}\right]$ & conv & convection \\
\hline & constant in ideal gas equation $\left[\mathrm{J} \mathrm{kg}^{-1} \mathrm{~K}^{-1}\right]$ & IR & thermal radiation \\
\hline $\mathrm{L}$ & absorber length $[\mathrm{m}]$ & $\mathrm{S}$ & solar radiation \\
\hline & fluid velocity $\left[\mathrm{m} \mathrm{s}^{-1}\right]$ & hc & heat conduction \\
\hline & inlet heat flux $\left[\mathrm{W} \mathrm{m}^{-2}\right.$ ] & $\mathrm{g}$ & gas \\
\hline $\mathrm{K}$ & permeability $\left[\mathrm{m}^{2}\right]$ & w & wall \\
\hline & specific isobaric heat & $\mathrm{Fa}$ & face \\
\hline & heat transfer coefficient of convective heat transfer & $\mathrm{F}$ & flow \\
\hline \multirow{3}{*}{$\begin{array}{l}\mathrm{k} \\
\mathrm{Q}_{\text {Abs.fl. }} \\
\mathrm{T}_{\text {outlet }}\end{array}$} & thermal conductivity $\left[\mathrm{W} \mathrm{m} \mathrm{m}^{-1} \mathrm{~K}^{-1}\right]$ & C & $\begin{array}{l}\text { channel } \\
\text { fluid }\end{array}$ \\
\hline & heat content in the air $[\mathrm{kJ} / \mathrm{kg}]$ & $\begin{array}{l}\mathrm{fl} \\
\mathrm{abs}\end{array}$ & $\begin{array}{l}\text { fluid } \\
\text { absorber material }\end{array}$ \\
\hline & outlet air temperature $[\mathrm{K}]$ & s & solid \\
\hline Greek s & mbols & por & porous structure \\
\hline
\end{tabular}

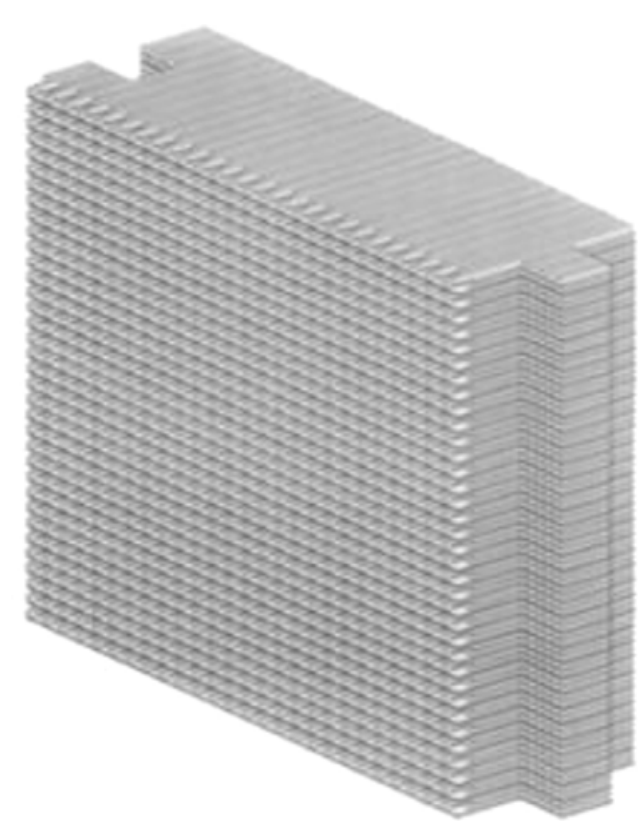

Fig. 1. SiSiC absorber module.
In 1990 a volumetric absorber made of $\mathrm{SiSiC}$ has been tested at the Plataforma Solar de Almeria (PSA) [8]. A picture of the concept absorber design is reported in Fig. 1.

In 1992 a new concept of selective receiver has been presented [9]. Placed before the inlet section of the ceramic receiver, a quartz glass structure was used as a selective medium, as shown in Fig. 2.

In 1996 Karni et al. [10] proposed the concept of a new volumetric absorber called the "Porcupine". A schematic view of this absorber is presented in Fig. 3.

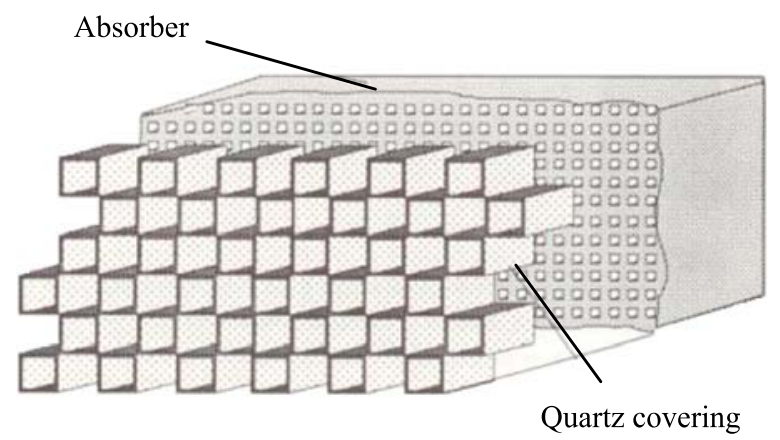

Fig. 2. Selective receiver concept. 

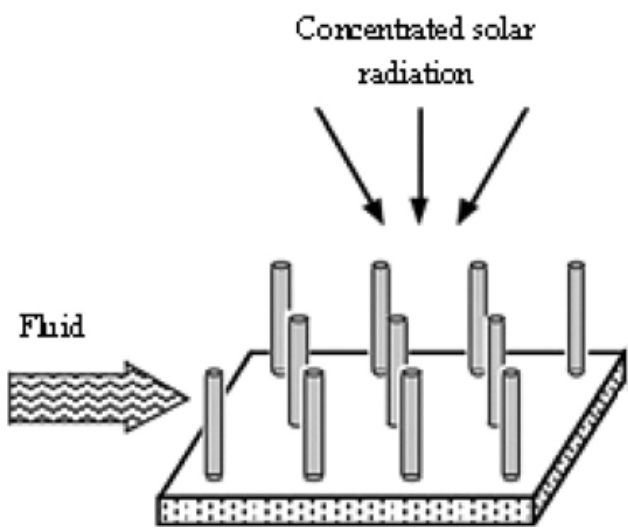

Fig. 3. The Porcupine absorber concept.

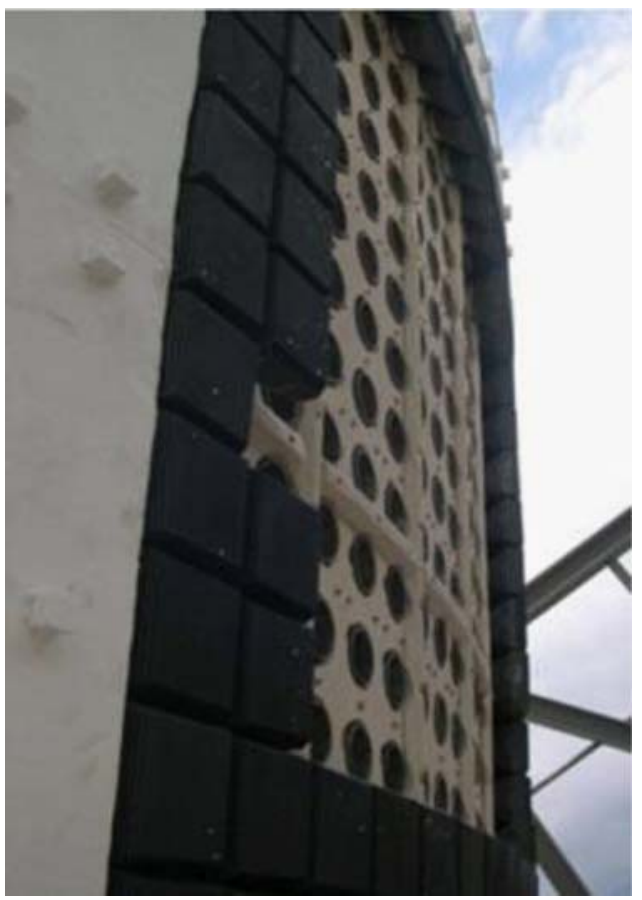

Fig. 4. SOLAIR 3000 during assembling.

In this directly-irradiated receiver, sunlight can penetrate and be absorbed along the elements enhancing the energy transport capability along each pin and among neighboring pins, preventing local overheating.

The High Temperature Receiver (HiTRec) was born in 1995 during the test of different ceramic materials in the DLR solar furnace in Cologne [11]. The receiver was composed of 37 modules; each module consisted of a hexagonal absorber structure and a SiSiC cup. The absorber honeycomb structure was made of recrystallized $\mathrm{SiC}$ with an open porosity of $49.5 \%$.

This concept has been improved in the past years until the SOLAIR 3000 showed in Fig. 4. This receiver concept mainly consists of modular ceramic absorbers with square-shape monoliths, a supporting structure and an air-return system. Using this technology, the Solar Tower of Jülich has been built in order to demonstrate this technology in a complete pre-commercial power plant for the first time.

In 2014, Ho et al. [12] presented in their work a review of central receiver designs for concentrating solar power applications. For each design, general principle and review of previous modeling and testing activities and research needs heve been reported.

Furthermore, in the latest work of Gupta et al. [13] a review of the solar power plants based on different solar concentrator systems have been presented, including central tower plants. The work gives an insight on the research being carried out worldwide on the thermodynamic performance evaluation of solar power generation systems, using different thermodynamic cycles.

Given the presence of different volumetric receivers, different numerical approaches have been developed in order to conduct a complete and accurate study of the performance of the latter.

Several modeling techniques have been used in order to adapt to different technologies; they are used both as design and analysis tool.

In 1990, Becker et al. [14] studied the heat exchange between the air and a ceramic structure characterized by square-section channel to be used in solar thermal applications using a monodimensional model. In 1991, Pitz-Paal et al. [9] evaluated the heat transfer efficiency between the air flow and a ceramic receiver with the presence of a transparent medium put before the receiver itself using a one-dimensional model that also took into account the radiative heat transfer. In 1996, Hoffschmidt et al. [7] also evaluated the heat transfer in a volumetric air receiver using a quasi-three dimensional simulation technique in which there was the presence of radial effective thermal conductivity. In 1998, Kribus et al. [15] studied the radiative heat transfer through a general participating media, considered as a homogeneous porous material where both solid and void zone cohexist, characterized by a porosity value $\varepsilon$.

This approach has been defined as continuum simulation procedure. Here, the inner structure of the porous medium is not defined, like in discrete representation, but it is characterized by a set of effective properties.

The same simulation approach has been taken up by several research groups, as it turned out to be very convenient from the

point of view of computational lightness and ability to simulate very complex internal structures.

Many studies have been conducted in order to have a proper characterization of effective properties in porous media used both in solar thermal and thermochemical applications. Petrasch et al. [16], Haussener et al. [17] evaluated effective properties of reticulated porous ceramics using tomography scan images of the material sample. Later on, Petrasch et al. [18] used the continuum approach for the characterization of the radiative heat transfer through a two-phase media. Caliot et al. [19], studied the heat exchange in reticulated porous ceramics to be used in solar thermal application.

In 2010, Smirnova et al. [20] compared the reults of the heat transfer analysis in a volumetric air receiver using both discrete and continuum simulation approaches.

In 2014, Kribus et al. [21] investigated the possible performance of volumetric absorbers as a function of geometric and material properties with the use of a simplified numerical model, aiming to identify the best absorber design parameters.

Róldan et al. [22] developed a two-dimensional simplified CFD model for the numerical characterization of ceramic and metallic absorbers under lab-scale conditions, considering thermal equilibrium between solid and fluid phases.

\section{Numerical models for advanced ceramic receivers}

The investigation of thermal performances of different volumetric air receivers was performed using diverse schematic representation of the receiver unit element, in case of discrete models, or outer shape, in case of continuum models. 


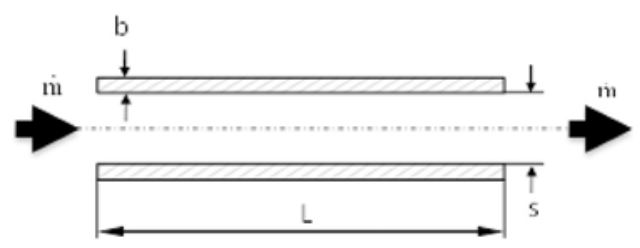

Fig. 5. Idealized one dimensional model.

\subsection{One-dimensional model developed by Becker et al.}

In 1990, Becker et al. [14] studied and predicted the behavior of a ceramic receiver through numerical simulation technique. In Fig. 5 the idealized one-dimensional model of a representative receiver channel used in the simulation is described. It was characterized by a square-type orifice of the channel and a length $(L)$ to width $(s)$ ratio initially equal to $10[L / s=10]$ and successively varied for prolongation of the channel $[L / s=25 ; L / s=100]$.

The wall thickness between adjacent channels was $b=3 \mathrm{~mm}$.

Then, the following physical data and models were used:

- Thermal conductivity $[k]=50 \mathrm{~W} / \mathrm{mK}$,

- Emissivity $[\varepsilon]=0.8 \mathrm{~A}$ standard air mass flow rate was defined as

$\dot{m}_{\mathrm{St}}=\dot{m} / A_{\mathrm{F}}=0.934 \mathrm{~kg} / \mathrm{m}^{2} \mathrm{~s}$

where $\dot{m}$ is the incoming mass flow rate and $A_{\mathrm{F}}$ is the cross section area. This value was chosen in order to heat up the air from $20^{\circ} \mathrm{C}$ to $1000{ }^{\circ} \mathrm{C}$ if all the incident radiation would have been transferred to the gas flow without any radiative losses.

Adiabatic boundary conditions were taken in the middle of the channel wall between adjacent channels

In this analysis the incident solar flux was considered constant and homogeneously distributed with a power of $100 \mathrm{~W} / \mathrm{cm}^{2}$. This value was assumed to be already reduced by direct reflection losses at the front surface.

The overall heat exchange within the model has been calculated by numerical treatment using the code LIW AK [23] calculating the temperatures of a system of nodes in any configuration. Then, the code ALBEMO [24] calculates transport factors to be used to describe heat transfer due to thermal radiation between the surface elements of a rectangular cavity.

The calculation is based on the Monte Carlo method. Several reflections at the walls of the cavity could take place between emission and absorption. The reflection is assumed to consist of a specular or a diffuse component with the choice of optional portions. The transport factors computed by ALBEMO code are used as input data for LIW-AK to calculate temperature distributions.

A correlation from tubes in cross flow by Churchill et al. [25] that covers the complete range of available data was chosen for the evaluation of the Nusselt number at the inlet area:

$\mathrm{Nu}=0.3+\frac{0.62 \cdot \sqrt{\operatorname{Re}} \cdot \sqrt{\operatorname{Pr}}}{\left[1+\left(\frac{0.4}{\mathrm{Pr}}\right)^{\frac{2}{3}}\right]^{\frac{3}{4}}} \cdot\left[1+\left(\frac{\mathrm{Re}}{28200}\right)^{\frac{5}{8}}\right]^{\frac{4}{5}}$

valid for a Reynolds number between $10^{2}$ and $10^{7}$ and a Péclet number higher than 0.2 .

The wall thickness of the channel has been used as the characteristic length $b$ in the Reynolds number calculation:

$\operatorname{Re}_{\mathrm{F}}=\frac{u \cdot b}{v}$

where $u$ is the fluid velocity in the channel and $v$ is the Kinematic viscosity. follow:

Furthermore, in Eq. (2), the Péclet number has been defined as

$\mathrm{Pe}=\frac{u \cdot b \cdot \rho \cdot c_{\mathrm{p}}}{k}$

where $\rho$ is the fluid density and $c_{\mathrm{p}}$ is the specific heat at constant pressure.

In the heat transfer coefficient correlation:

$\mathrm{Nu}=h \cdot \frac{b}{k}$

the flow in the channel was considered as developing flow, which means slug flow at the entrance changing into laminar flow, at uniform wall temperature. For this case, Kays [26] derived the following empirical correlation:

$\mathrm{Nu}_{\mathrm{C}}=3.66+\frac{0.104 \cdot \frac{\mathrm{s}}{\mathrm{x}} \cdot \operatorname{Re}_{\mathrm{C}} \cdot \operatorname{Pr}}{1+0.016 \cdot\left[\frac{\mathrm{s}}{\mathrm{x}} \cdot \operatorname{Re}_{\mathrm{C}} \cdot \operatorname{Pr}\right]^{0.8}}$

Here, the Prandtl number is defined as:

$\operatorname{Pr}=\frac{c_{p} \cdot \mu}{k}$

where $\mu$ is the dynamic viscosity. Moreover, in Eq. (5), the hydraulic diameter of a square cross-section channel $s$ and the distance along the channel $\mathrm{x}$ has been introduced already:

$\operatorname{Re}_{\mathrm{C}}=\frac{u_{c} \cdot s}{v}$

$\mathrm{Nu}_{\mathrm{C}}=\mathrm{h}_{\mathrm{C}} \cdot \frac{s}{k}$

To account for temperature dependent physical properties of the heated air, a correction factor is applied on the preceding Nusselt number correlations:

$\left(\frac{T_{g}}{T_{W}}\right)^{0.45}$

with: $0.5<\frac{\mathrm{T}_{\mathrm{g}}}{\mathrm{T}_{\mathrm{W}}}<1.5$

in corresponding sections of gas flow and channel wall.

The flow through the receiver itself is represented by a model that considers the pressure drop $\left(\Delta p_{\text {rec }}\right)$ as composed by three different terms: one for the losses into the inlet section, one for the losses into the outlet section and the last one for the friction term along the channel walls:

$\Delta p_{\text {rec }}=\left(\zeta_{1, \text { in }}+\zeta_{1, \text { out }}+\zeta_{\mathrm{f}}\right) \frac{1}{2}\left(\frac{\dot{m}}{A}\right)^{2}$

where:

$\zeta_{1, \text { in }}=\left[1.5-\left(\frac{A_{\mathrm{o}}}{A_{\mathrm{Fa}}}\right)\right] \frac{1}{\rho_{\text {in }}}$

$\zeta_{\text {l,out }}=\left[2 \frac{A_{\mathrm{o}}}{A_{\mathrm{F}}}\left(\frac{A_{\mathrm{o}}}{A_{\mathrm{F}}}-1\right)\right] \frac{1}{\rho_{\text {out }}}$

$\zeta_{\mathrm{f}}=\frac{64}{1.13 \cdot \operatorname{Re}} \frac{L}{s} \frac{1}{\rho_{\mathrm{m}}}$

where $\rho_{\text {in }}, \rho_{\text {out }}$ and $\rho_{\mathrm{m}}$ are the fluid densities at the inlet section, the outlet face and in the middle of the channel respectively.

3.2. One-channel receiver model for a selective solar receiver developed by Pitz-Paal

An advanced volumetric solar receiver concept has been presented in 1991 by Pitz-Paal et al. [9] It consists of a ceramic receiver, characterized by straight square-section channels, covered by a matrix of square channels of quartz transparent glass. In order to quantify the efficiency improvements for such a receiver, 


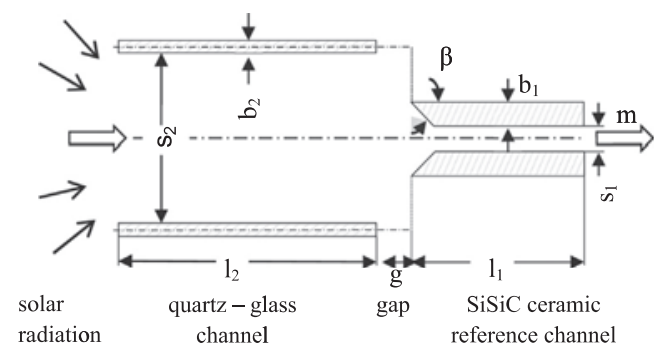

Fig. 6. The one-channel selective receiver model.

a simplified one-channel receiver model, showed in Fig. 6, has been developed. Here, the section of the quartz square channel is placed before the one characterizing the ceramic structure. The transparent zone is defined through the wall thickness $b_{2}$, the hydraulic diameter $s_{2}$ and the length $L_{2}$. In addition, the ceramic zone is defined through the wall thickness $b_{1}$, the hydraulic diameter $s_{1}$ and the length $L_{1}$ and between the two zones, a gap $g_{2}$ is present.

Interaction between adjacent channels has been considered by symmetric assumptions:

1. Concentrated solar radiation has symmetric distribution, corresponding to the channel axis;

2. Wall strength of quartz glass is negligible;

3. Reflection on glass wall is specular exclusively;

4. Interaction of the thermal radiation between quartz glass and ceramic section is considered by defining an average ceramic front temperature through the temperature distribution in the reference ceramic channel.

From these assumptions follows that the reflected and transmitted radiation can be assumed to be reflected at the quartz wall with a reflectivity that is the sum of material reflectivity and absorptivity.

The proposed model was unable to show the efficiency improvements of the whole receiver due to the neglect of boundary effects and inhomogeneity of the solar input over the inlet section. Nevertheless, it showed the potential for efficiency improvements by the quartz glass structure and limits of the absorber temperatures [9].

The one-channel receiver model considered finite wall elements which were assumed to be isothermal. The steady state energy equation is then applied to each element:

$q_{\text {conv }} A_{\mathrm{f}}+q_{\mathrm{ir}} A_{\mathrm{f}}+q_{\mathrm{s}} A_{\mathrm{f}}+q_{\mathrm{hc}} A_{\mathrm{hc}}=0$

The single energy transfer mechanisms incorporated in this equation are calculated as follows:

1. Heat transfer by thermal radiation

$\mathrm{q}_{\mathrm{ir}}=\sum_{\mathrm{k}} \mathrm{a}_{\mathrm{ik}} \mathrm{T}_{\mathrm{wk}}^{4}$

where $T_{\mathrm{w}}$ is the wall temperature and $a_{\mathrm{i}}$ derived from the enclosure method [27], extended to specular reflected surfaces.

1. Heat transfer by solar radiation

$\mathrm{q}_{\mathrm{s}}$ independent from $T_{\mathrm{w}}$

calculated by Monte-Carlo ray-tracing algorithm

2. Heat transfer by conduction

$q_{\mathrm{hc}}=-k \Delta T_{\mathrm{w}}$

as a linearization of Fick's law, where $k$ is the heat conductivity of the material and $\Delta T_{\mathrm{w}}$ is the temperature difference at the wall.

\section{Heat transfer by convection}

$q_{\mathrm{conv}}=h\left(T_{\mathrm{g}}-T_{\mathrm{w}}\right)$

from Navier-Stokes simulation under constant wall temperature assumption [28] defining $h$ as the convective heat transfer coefficient of the fluid.

Also the gas flow is divided into finite volume elements. In case of constant specific heat, the steady state energy equation is:

$\dot{M} c_{\mathrm{p}} \Delta T_{g}^{j}=\sum q_{\text {conv }}^{i} A_{\mathrm{v}}^{i}$

where $i$ and $j$ are the index of the elements considered, $c_{\mathrm{p}}$ is the specific heat with fixed pressure of the fluid and $A_{\mathrm{v}}$ is the heat transfer surface per volume.

Making reference to Fig. 6, different non-dimensional parameters, necessary for the characterization of the convective heat transfer, have been evaluated. The required Reynolds and local Nusselt number for the quartz-glass channel (Zone 1) have been calculated as:

$\operatorname{Re}_{1}=\frac{\dot{m} s_{1}}{\Delta_{1} \mu t_{\mathrm{g}}}$

$\mathrm{Nu}_{1}=\mathrm{Nu}\left(\gamma \cdot x^{*}, \operatorname{Re}_{1}, \operatorname{Pr}\right)$

where $m$ is the mass flow density of the heat transfer fluid, $\mu$ is its dynamic viscosity, $\Delta_{1}$ is the porosity of the quartz cover defined as $\frac{s_{1}^{2}}{\left(b_{1}+s_{1}\right)^{2}}, t_{\mathrm{g}}$ is the non-dimensional temperature defined as the ratio between the gas entrance temperature and the ambient temperature, $\gamma$ is the ratio of the absorber hydraulic diameter and the quartz cover diameter and $x^{*}$ is defined as the ratio between the spatial coordinate $x$ and $s_{2}$.

For the ceramic receiver channel (Zone 2), the following Reynolds number and Nusselt number correlations have been used:

$\operatorname{Re}_{2}=\frac{\dot{m} s_{2}}{\Delta_{2} \mu t_{\mathrm{g}}}$

$\mathrm{Nu}_{1}=\mathrm{Nu}\left(x^{*}-\left(l_{1}^{*}+d^{*}\right) / \gamma, \operatorname{Re}_{2}, \operatorname{Pr}\right)$

where $\Delta_{2}$ is the porosity of the ceramic reference channel defined as $\frac{s_{2}^{2}}{\left(b_{2}+s_{2}\right)^{2}}, l_{1}^{*}$ is the ratio between $l_{1}$ and $s_{1}$ and $d^{*}$ is the ratio between $d$ and $s_{2}$.

3.3. Quasi-three-dimensional analytical model for a volumetric absorber structure developed by Hoffschmidt

The numerical models presented so far are based on onedimensional approaches. The model presented herein is instead defined as quasi-three-dimensional introducing the effective radial heat conductivity $k_{\text {eff }}$ that includes effects of conduction and thermal radiation [7].

The model of the volumetric receiver is here approached by a discretization of the absorber into small parallel sections. It can

be assumed that each of these sections is irradiated homogeneously. The geometry of the receiver is shown in Fig. 7, characterized by a circular cross section.

The temperature distribution of each section will be evaluated by

a one-dimensional approach, which is extended by terms to introduce the coupling between those parallel sections.

The steady-state energy balance for the absorber material is given by:

$A_{\mathrm{v}} \alpha(z)\left[T_{\mathrm{fl}}(z)-T_{\mathrm{abs}}(z)\right]+(1-\epsilon) k \frac{\mathrm{d}^{2} T_{\mathrm{abs}}}{\mathrm{d} z^{2}}-2 \pi \sum_{i=1}^{N} w_{\mathrm{i}} \mu_{\mathrm{i}} \frac{\mathrm{d} I_{\mathrm{i}}}{\mathrm{d} z}+\Omega_{r_{\varphi}}(z)=0$ 


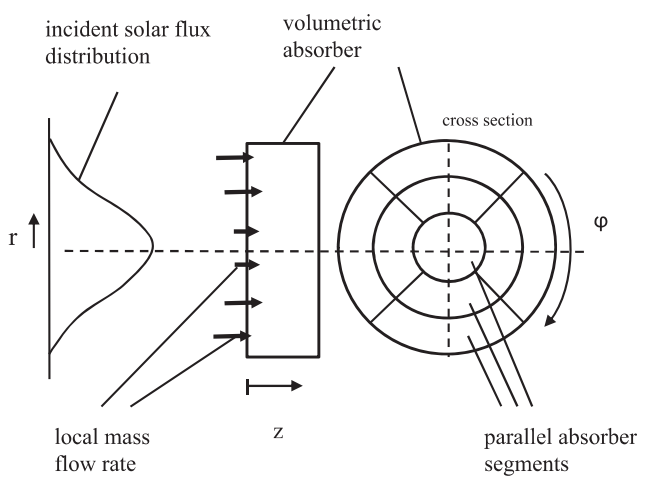

Fig. 7. Geometry of the receiver model.

It balances the convective heat transfer, the axial heat conduction, the absorption of the radiation intensity (integrated over all different directions) and the source term $\Omega_{r_{\varphi}}$ describing the effects of radial heat transport. This term is evaluated as follows:

$\Omega_{r \varphi}=\frac{1}{r} \frac{\partial}{\partial r}\left(r k_{\mathrm{eff}} \frac{\partial T_{\mathrm{abs}}}{\partial r}\right)+\frac{1}{r} \frac{\partial}{\partial r}\left(\frac{k_{\mathrm{eff}}}{r} \frac{\partial T_{\mathrm{abs}}}{\partial \varphi}\right)$

The effective radial heat conductivity $k_{\text {eff }}$ includes effects of conduction and thermal radiation.

The steady state energy balance of the fluid is given by:

$\dot{m} c_{\mathrm{p}} \frac{\mathrm{d} T_{\mathrm{fl}}}{\mathrm{d} z}=h(z) A_{\mathrm{v}}\left[T_{\mathrm{abs}}(z)-T_{\mathrm{fl}}(z)\right]$

where $A_{\mathrm{v}}$ is the heat transfer surface per volume. It is assumed that no radial mass transfer occurs. This assumption strictly applies only to honeycomb structures because the channel structure prevents radial mass flow. However, good agreement between experimental and numerical data, obtained through the use of this model, for other porous materials, such as different honeycomb structures, wire mesh, etc.; suggests that the effects of radial flow are negligible in the presented application.

The pressure loss characteristic is described by the Forchheimer extension to Darcy's law:

$\frac{\partial p}{\partial z}=a \mu_{\mathrm{fl}} u+b \rho_{\mathrm{fl}} u^{2}$

The coefficients $a$ and $b$ are characteristic for the absorber geometry and are determined by measurement of the pressure loss at ambient temperature as reported in Hoffschmidt.

The total mass flow rate which is given as a boundary condition is evaluated by integration of the mass flow density over the absorber cross-section:

$\dot{M}_{\mathrm{fl}}=\iint_{\mathrm{A}} \dot{m} \mathrm{~d} A$

The convective heat transfer coefficient is evaluated from the following correlation:

$\mathrm{Nu} A_{\mathrm{v}}=c \operatorname{Re}^{m}$

where the definition of the heat transfer surface per volume $A_{\mathrm{v}}$ and the coefficient $c$ have been defined by Hoffschmidt.

The discrete ordinate method and the axial temperature distribution are used to solve this set of equations using a finite difference scheme. The alternate application of the two solvers using the respective results of absorbed intensity and axial temperature leads to a convergent solution for each absorber segment.

3.4. Discrete and continuum approach for the investigation of flow through volumetric receiver developed by Fend and Smirnova

The models presented in the previous paragraphs were based on the idealized representation of a small section of the absorber material used in open volumetric receivers. On the other hand, the investigation on the entire structure of the receiver leads to a better prediction of its overall performance and its behavior.

For this purpose, two numerical models have been developed, both characterized by a three-dimensional representation. The first one uses the real geometry of the channel (discrete model), while the second one is characterized by a homogeneous representation of the absorber volume (continuum model).

In this study, the absorbed concentrated solar radiation has been considered as a volumetric heat source described by an exponential law [29]:

$I=I_{0} \cdot \mathrm{e}^{-\xi \cdot z}$

The heat flux $I_{0}$ used for the simulation was in accordance with the average experimental value and the extinction coefficient $\xi$ has been found experimentally through the transmittance measurements of different investigated samples.

Furthermore, because of the full symmetry of the geometry in the single channel as well as in the whole receiver module, in both cases only one quarter of the volume has been used for the analysis.

\section{Single-channel model}

For the numerical calculation of the velocity and pressure fields the Navier-Stokes application mode has been used in the model. This application mode describes the connection of the fluid velocity and the pressure according to the equation:

$\mu \cdot \nabla\left(\nabla u+(\nabla u)^{T}\right)=\rho \cdot u \cdot \nabla u$

where the exponent $T$ refers to the fluid temperature and:

$\nabla(\rho \cdot u)=0 ; \rho=\rho\left(p, T_{\mathrm{fl}}\right)$

The convective heat transfer and the heat conduction in the air are simulated through the next equation:

$\nabla\left(-\lambda \cdot \nabla T_{\mathrm{fl}}+\rho \cdot c_{\mathrm{p}} \cdot u \cdot T_{\mathrm{fl}}\right)=Q$

while the heat conduction in the solid body is defined by the following equation:

$-\nabla\left(\lambda \cdot \nabla T_{\mathrm{s}}\right)=\int_{0}^{z} q_{0}$

where:

$q_{0}=Q_{\mathrm{abs}} \cdot \frac{\xi \cdot \mathrm{e}^{(-\xi \cdot z)}}{A_{\text {in }}}$

Here, $q_{0}$ is the heat source term and $Q_{a b s}$ is the absorbed heating power from the solid body.

\section{Continuum model}

The continuum model presented herein considers the volume of the entire receiver as a porous continuum medium with homogenized properties, either experimentally evaluated (such as the permeability or the effective heat conductivity) or derived from the single-channel model.

This model includes the heat transfer from the hot face of the solid body to the air flow by taking into account the volumetric heat transfer coefficient, an experimentally determined quantity.

For the numerical evaluation of the velocity and pressure fields also for the continuum approach the Navier-Stokes equations have been used. Furthermore, since it has been treated as a porous medium, the area of the receiver has been described by a slight modification of the Navier-Stokes equation, the Brinkman equation:

$\left(\frac{1}{\epsilon}\right) \mu \cdot \nabla\left(\nabla u+(\nabla u)^{T_{\mathrm{fl}}}\right)=\left(\frac{\mu}{K}\right) \cdot u$ 

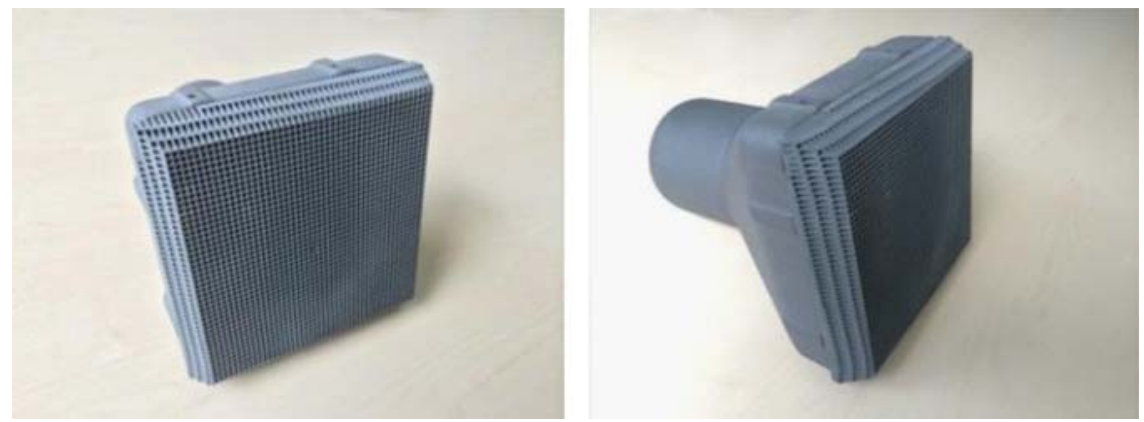

Fig. 8. HiTRec-II modular single cup.

The heat transfer in the air has been simulated according to the following equation:

$\nabla\left(-\lambda \cdot \nabla T_{\mathrm{fl}}+\rho \cdot c_{\mathrm{p}} \cdot u \cdot T_{\mathrm{fl}}\right)=Q$

The heating power per unit volume $Q$ has been determined through the so called volumetric heat transfer coefficient $h A_{v}$ [W $\mathrm{m}^{-3} \mathrm{~K}^{-1}$ ] according to the following equation:

$Q=h \cdot A_{\mathrm{v}} \cdot\left(T_{\mathrm{s}}-T_{\mathrm{fl}}\right)$

The volumetric heat transfer coefficient $h A_{v}$ has been calculated according to the equation:

$\mathrm{Nu} A_{\mathrm{v}}=c \mathrm{Re}^{m}$

Thus, the volumetric term $h A_{\mathrm{v}}$ has been evaluated from the Nusselt number correlation:

$h \cdot A_{\mathrm{v}}=\frac{\mathrm{Nu} \cdot A_{\mathrm{v}} \cdot k}{\delta}$

where $\delta$ was the characteristic length of the pore structure, which was in this case the channel diameter.

The heat transfer in the solid body has been simulated through the heat conduction module, based on the following equation:

$-\nabla\left(k_{\text {por }} \cdot \nabla T_{s}\right)=q_{0}$

where $q_{0}$ is the heating power per unit volume (heat source term) that takes into account the absorption of the solar radiation in the receiver as well as the heat transferred to the fluid. $k_{\text {por }}$ is the heat conductivity in the porous structure, which has been determined experimentally as a function of temperature:

$k_{\text {por }}=(1-\epsilon) \cdot 280.7 \cdot e^{\left(-0.0021 \cdot T_{s}\right)}$

The complete equation for the heat source term is then represented by:

$q_{0}=I_{0} \cdot \epsilon \cdot \xi \cdot \mathrm{e}^{-\xi \cdot z}+h \cdot A_{\mathrm{v}} \cdot\left(T_{\mathrm{s}}-T_{\mathrm{fl}}\right)$

\section{Methodology of validation - experimental set-up}

To investigate performance characteristics of the receiver, a test-bed, able to supply boundary conditions similar to the application in the power plant for a receiver module, has been used [29].

The concentrated radiation meets the inlet surface of the receiver sample and heats it up. The blower supplies the pressure difference and causes an air flow through the sample. Thus cold ambient air is heated up to defined temperatures, which are measured with thermocouples at several points.

For the purpose of the current study, no return air flow was used. Besides air and water temperatures also flow rates of water and air are measured and logged.

Additionally, radiation flux and surface temperature are measured with separate devices. For the radiation flux, the camera- target system FATMES is employed [30]. Surface temperature has been monitored with standard infrared thermography.

Efficiency is calculated according to equation:

$\eta=\frac{Q_{\text {Abs.exp. }}}{Q_{\text {Radiation }}}$

Here, $Q_{\text {Radiation }}$ is the radiative concentrated power from the lamps as measured by FATMES, $Q_{\text {Abs.exp. }}$ is the measured heat content in the air:

$Q_{\text {Abs.exp. }}=\dot{M} \cdot c_{p} \cdot\left(T_{\text {outlet }}-T_{\text {inlet }}\right)$

Efficiency and outlet air temperature are the key-parameters, which are used for the comparison of the different numerical results.

\section{Results and comparison}

In this paragraph, simulation results of the numerical models analysed are reported and compared with measured values, which represent the reference case used for the results comparison [31], except for the model proposed by Becker et al. for which it was not possible to reset the model by referring to the reference case.

Key parameter for the comparison will be the air temperature, evaluated at the outlet section, and the efficiency, defined as the

ratio between the effective power transmitted to the fluid and the irradiative concentrated power.

All the numerical models have been set using initial, boundary and environmental conditions taken from the experimental analysis settings.

Furthermore, the Hoffschmidt model has been used in one dimensional mode to match the reference case.

Thus, the main irradiation value has been set referring to the one developed from the experimental setup and equal to $652,800 \mathrm{~W} / \mathrm{m}^{2}$; the mass flow is set referring to the value measured from the experiment and equal to $0.0024 \mathrm{~kg} / \mathrm{s}$. As evolving fluid, moist air with $5 \%$ relative water content (evaluated at $285.15 \mathrm{~K}$ and $1.021 \mathrm{bar}$ ) has been used during the simulation.

The geometry considered during the simulations refers to the current volumetric receivers state-of-the-art, the HiTRec-II, latest evolution of the High Temperature Receiver (HiTRec) developed by DLR. In particular, the geometry object of the study, reported in Fig. 8, presents finer walls and the characteristic square shape for each modular element.

As it is shown from the temperature profiles reported in Fig. 9, the discrete numerical models (Pitz-Paal, Hoffschmidt, Fend $\mathcal{E}$ Smirnova - single channel) well reproduce the heat transfer between solid and fluid phase in the volumetric receiver. The curves are easily stackable and also a good agreement is shown regarding the corresponding depth of the control volume in which solid and fluid phase reach the thermal equilibrium. 


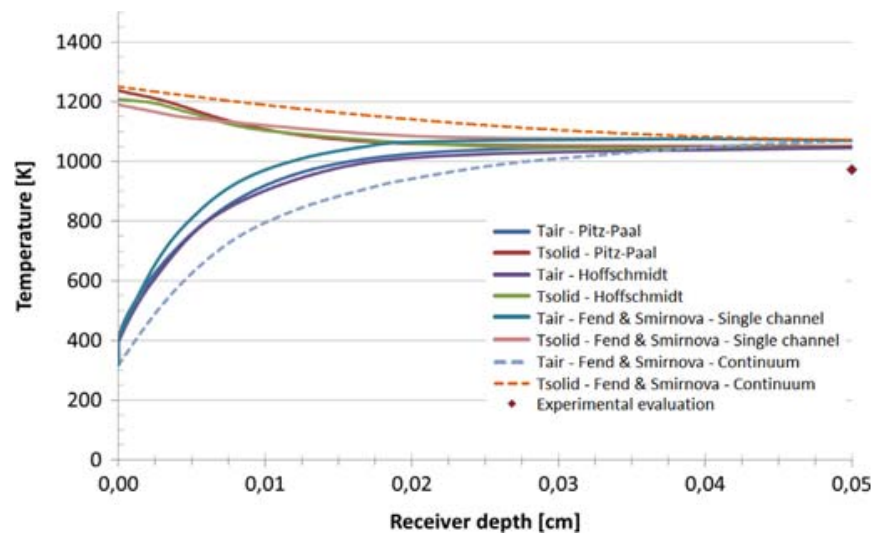

Fig. 9. Mean temperature profiles of solid and fluid phase.

Table 1

\begin{tabular}{llll}
\hline & $T_{\text {outlet }}[\mathrm{K}]$ & $Q_{\text {Abs.fl. }}[\mathrm{W}]$ & $\eta$ \\
\hline Pitz-Paal model & 1050 & 1.90 & 0.78 \\
Hoffschmidt model & 1048 & 1.89 & 0.77 \\
Fend \& Smirnova - single channel & 1057 & 1.92 & 0.79 \\
Fend \& Smirnova -continuum & 1073 & 1.96 & 0.81 \\
Experimental evaluation & 972 & 1.69 & 0.69 \\
\hline
\end{tabular}

The only continuum approach represented on the graph (Fend $\mathcal{E}$ Smirnova - continuum) still shows a good agreement concerning the temperatures at the ends of the curves but, in this particular case, the thermal equilibrium is reached in deepest point of the volumetric receiver. This is due to the homogeneous representation of the porous media that is less accurate than the discrete implementation of the volume control, in which fluid zone and solid area appear to be clearly separated.

On the same graph, the measured air temperature is reported, pointing out a gap with the predicted numerical values.

The results are summarized in Table 1.

The outlet temperature of the air, as well as the power absorbed by the fluid and the thermal efficiency of the discrete numerical models, are comparable and all of them overestimated the performance of the receiver compared to the measured value. The values regarding the continuum simulation are even higher compared to the discrete ones, this is due to the approximation introduced from the continuum approach itself.

The difference between the expected numerical results and the experimental evaluation, may be due to the inability of these models to effectively represent the phenomenon of radiative exchange within the porous medium considered. In the numerical models mentioned above, the radiation was considered as a phenomenon incorporated in the pre-existing conductive heat exchange model. In this case, it was not possible to obtain a good representation of the losses linked to the radiation that was not absorbed within the volume control but, instead, was reflected or re-emitted to the outside through emission and back-scattering phenomena.

Furthermore, variable environmental conditions and the difficulty in the experimental evaluation of the air flow mean temperature, also influenced the final measured value. In this particular case, thermocouples have been used for this analysis and they have been placed on the back of the outflow zone of the receiver in proximity of the hot gas tube. In this case, losses regarding the flow of hot air may occur and they are difficult to assess. For this reason the evaluation of the temperature of the air flow output can be affected by such uncertainty evaluation.

\section{Conclusion}

In order to develop new high-performing structures, suitable numerical simulation approaches are necessary in order to achieve an adequate prediction of the energy behavior of the latter.

In the present work, an overview regarding the latest developments of volumetric solar receiver and the in-house numerical approaches useful for the characterization of their thermo-fluid dynamic behavior has been presented and discussed.

The numerical models presented in this work show an overestimation of the performances of the volumetric receiver object of study. This is mainly due to variable environmental conditions, difficulty in the experimental evaluation of the air flow mean temperature and the difficulty of all the models in the representation of the radiative heat exchange. This condition becomes even more obvious in the case of the continuum approach presented by Fend \& Smirnova where, additionally, the inherent approximation of the homogeneous representation is present.

One of the biggest limitations of discrete models concerns the heaviness of calculation due to the necessity to use a detailed representation of the porous volume. In this regard, the development of a numerical model based on the continuum approach, can be effective as it is released from the detailed representation of the porous medium.

Furthermore, a better implementation of the radiative exchange within the volume control is required. This can be achieved setting a multiphysic numerical simulation where the radiation is treated as a stand-alone physic and, at the same time, coupled with convection and conduction physics, obtaining more reliable results. An advisable option can be to use the continuum model for the design of new structures, having the possibility to easily and quickly manipulate the geometric and thermodynamic parameters of the receiver. Once the new geometry is defined, a multiphysic discrete simulation can be set in order to have a more reliable numerical prediction of the receiver performance.

\section{Acknowledgments}

This work was carried out with financial support from the Ministry of Innovation, Science and Research of the State of North Rhine-Westphalia (MIWF NRW), Germany under contract 3232010-006 (Start-SF).

\section{References}

[1] Sizmann RL. Solar radiation conversion. In: Solar power plants. Fundamentals, technology, systems, economics, Berlin: Springer-Verlag; 1991. p. 17-83.

[2] DeMeo EA, Galdo JF. Renewable energy technology characterizations. Topical report. Palo Alto, CA: US DOE-Washington and EPRI; 1997.

[3] Falcone PK. A handbook for solar central receiver design. Livermore, CA: Sandia National Laboratories; 1986 SAND 86-8009.

[4] Grasse W, Hertlein HP, Winter CJ. Thermal solar power plants experience. In: Vant-Hull LL, editor. Solar power plants. Fundamentals, technology, systems, economics. Berlin: Springer-Verlag; 1991. p. 215.

[5] Mancini TR, Kolb GJ, Prairie M. Solar thermal power. In: Boer KW, editor Advances in solar energy: an annual review of research and development. Boulder, CO: American Solar Energy Society; 1997. p. 1-42.

[6] Hennecke K, Schwarzbözl P, Alexopoulos S, Hoffschmidt B, Göttsche J, Koll G, Beuter M, Hartz T. Solar power tower Jülich - the first test and demonstration plant for open volumetric receiver technology in Germany. In: Proceedings of the 14th Biennial CSP Solar PACES Symposium, USA: Las Vegas; March 4-7, 2008.

[7] Hoffschmidt B. Vergleichende Bewertung verschiedener Konzepte volumetrischer Strahlungsempfänger (Thesis). Aachen, Germany: RWTH; 1996.

[8] Ávila-Marín AL. Volumetric receivers in solar thermal power plants with central receiver system technology: a review. Solar Energy 2011;85:891-910.

[9] Pitz-Paal R. Entwicklung eines selektiven, volumetrischen receivers fuer Solarturmkraftwerke-Parameter-Untersuchungen und exergetische Bewertung (Dissertation). . DLR; 1993. p. 163S Forschungsbericht. 
[10] Karni J, Kribus A, Rubin R, Doron P. The "Porcupine": a novel high-flux absorber for volumetric solar receivers. J Solar Energy Eng Trans ASME 1998;120(2):85-95.

[11] Hoffschmidt B, Fernandez V, Konstandopoulos AG, Mavroidis I, Romero M, Stobbe P, Te'llez F, Development of ceramic volumetric receiver technology. In: Proceedings of 5th Cologne Solar Symposium. Forschungsbericht 10. Germany: DLR; 2001, p. 51-61.

[12] Ho CK, Iverson BD. Review of high-temperature central receiver designs for concentrating solar power. Renew Sustain Energy Rev 2014;29:835-46.

[13] Gupta MK, Kaushik SC, Ranjan KR, Panwar NL, Siva Reddy V, Tyagi SK. Thermodynamic performance evaluation of solar and other thermal power generation systems: a review. Renew Sustain Energy Rev 2015;50:567-82.

[14] Becker M, Böhmer M, Cordes S. DLR/Ceramtec Volumetric ceramic foil receiver. SSPS Technical report no.1/91; 1991, p. 11-14.

[15] Kribus A, Ben-Zvi R, Fiterman A. A numerical simulation method for radiative transport in general participating media. In: Proceeding of the 27th Israel Conference on Mechanical Engineering; 1998

[16] Petrasch J, Schrader B, Wyss P, Steinfeld A. Tomography-based determination of the effective thermal conductivity of fluid-saturated reticulate porous ceramics. J Heat Transf 2008;130(3):032602.

[17] Haussener S, Steinfeld A. Tomography-based heat and mass transport characterization of complex porous materials for solar power and fuel generation. World Engineers Convention; 2011

[18] Petrasch J, Haussener S, Lipinski W. Discrete vs. continuum-scale simulation of radiative transfer in semitransparent two-phase media. J Quant Spectrosc Radiat Transf 2011:112(9):1450-9.

[19] Caliot C, Wu Z, Flamant G, Wang Z. Numerical simulation of convective heat transfer between air flow and ceramic foams to optimise volumetric solar air receiver performances. Int J Heat Mass Transf 2011;54(7-8):1527-37.

[20] Smirnova O, Fend T, Schwarzbözl P. Schöllgen D. Homogeneous and Inhomogeneous Model for Flow and Heat Transfer in Porous Materials as High Temperature Solar Air Receiver. Proceedings of the European COMSOL Conference; 2010.
[21] Kribus A, Grijnevich A, Gray M, Caliot Y. Parametric study of volumetric absorber performance. Energy Procedia 2014:49:408-17.

[22] Roldán MI, Avila-Marin A, Alvarez-Lara M, Fernandez-Reche J. Experimental and numerical characterization of ceramic and metallic absorbers under labscale conditions. Energy Procedia 2015;69:523-31.

[23] Grönefeld G. Program LIW AK Interatom-Notiz. Nr 35.02353.3; 1985.

[24] Müller K. ALBEMO, ein Programm zur Berechnung des Strahlungstransportes in Hohlräumen fur Neutronen-, Gamma- und Wärmestrahlung InteratomBericht. Nr. 70.03565.6; 1985.

[25] Churchill SW, Bernstein M, Correlating A. Equation for forced convection from gases and liquids to a circular cylinder in crossflow. Heat Transfer 1977:99:300-6.

[26] Kays WM. Trans ASME 1955;77:1265.

[27] Siegel R, Howell JR. Radiation heat transfer. Washington: MacGraw Hill Book Company; $1981 / 82$

[28] Freudenstein K, Karnowsky B, Reinders R. Evaluation of Convective Heat Coefficients and Comparison with Semiempirical Correlations in a Volumetric Ceramic Receiver. Studie für DLR Köln in DLR ET-1/90-2.

[29] Fend T, Hoffschmidt B, Pitz-Paal R, Reutter O. Cellular ceramics use in solar radiation conversion. In: Scheffer M, Colombo P, editors. Cellular ceramics: structure, manufacturing and applications. Weinheim: Willey-VCH GmbH \& Co. $\mathrm{KgA} ; 2005$.

[30] Kalt A, Becker M, Dibowski G, Groer U, Neumann A. The New Solar Furnace of the DLR, Köln, Germany, Specifications and first test results. In: Proceedings of the 7th International Symposium on Solar Thermal Concentrating Technologies. Moscow; September 26-30, 1994, p. 1023-1035.

[31] Fend T, Hoffschmidt B, Pitz-Paal R, Reutter O, Rietbrock P. Porous materials as open volumetric solar receivers: Experimental determination of thermophysical and heat transfer properties. Energy 2004;29(5-6):823-33 SolarPACES 2002. 